\title{
CASOS DE MALFORMACIONES EN TRILOBITES DE ESPAÑA
}

\author{
Isabel RÁBANO' y Miguel ARBIZU \\ ' Museo Geominero, ITGE, Ríos Rosas 23, 28003 Madrid. \\ - Departamento de Geología, Facultad de Geología. Universidad de Oviedo, \\ Jesús Arias de Velasco s/n, 33005 Oviedo.
}

Rábano, I. y Arbizu, M. 1999. Casos de malformaciones en trilobites de España. [Exoskeletal abnormalities in trilobites from Spain]. Revista Española de Paleontología, $\mathbf{n}^{\circ}$ extr. Homenaje al Prof. J. Truyols, 109-113. ISSN 0213-6937.

\begin{abstract}
In the last years attention has been paid to the study of trilobite malformations by numerous paleontologists having even done some synthetic work on this subject. Up to now four of these malformation cases have been recorded in the Iberian Peninsula and only one of them, Placoparia (Coplacoparia) borni Hammann, is included in the most complete synthetic work on this subject which has been written by Owen in 1985. The present paper shows three cases of malformations in Spanish trilobites; two of them are new whereas the third one corresponds to a previous quotation from Smeenk in 1983. A cephalon of Asaphellus toledanus (Gil Cid), from the Middle Ordovician of Navas de Estena (province of Ciudad Real), shows a healed injury on the right librigena and genal point; the wound was most probably caused during a sublethal attack of a durophagous predator. Two pygidia of Platyscutellum castroi (Mallada), from the Lower Devonian of Colle (province of León), show an unusual ribarchitecture, most probably due to activity of parasites, which affected the pygidia in different degrees.
\end{abstract}

Keywords: Trilobita, exoskeletal abnormalities, Ordovician, Devonian, Spain.

\section{RESUMEN}

En los últimos años, el estudio de malformaciones en trilobites ha suscitado el interés de numerosos paleontólogos, habiéndose realizado incluso algún trabajo de síntesis sobre este tema. En la Península Ibérica se han registrado hasta el presente cuatro casos de estas malformaciones y unicamente uno de ellos, Placoparia (Coplacoparia) borni Hammann, se recoge en el trabajo de síntesis más completo sobre este tema, que ha sido realizado por Owen en 1985. En el presente estudio se presentan tres casos de malformaciones en trilobites españoles, dos de los cuales son novedad, mientras que el otro corresponde a una de las citas anteriormente existentes y realizada por Smeenk en 1983. El primero de estos casos consiste en una regeneración posttraumática de la librigena y punta genal derecha del asáfido Asaphellus toledanus (Gil Cid), probablemente originaḍa durante el ataque de un predador. El ejemplar procede del Ordovícico Medio de Navas de Estena (Ciudad Real). Los dos casos restantes se atribuyen a parásitos que afectaron en distintos grados a sendos pigidios del scutélido Platyscutellum castroi (Mallada), procedentes del Devónico Inferior de Colle (León).

Palabras clave: Trilobites, malformaciones exoesqueléticas, Ordovícico, Devónico, España.

\section{INTRODUCCIÓN}

El registro de malformaciones en las piezas que constituyen el exoesqueleto dorsal de los trilobites ha sido tratado con amplitud en la literatura paleontológica (referencias compiladas en Owen, 1985 y Babcock, 1993). Estas malformaciones han sido atribuidas a tres causas (Owen, 1985): a) Lesiones traumáticas, provocadas generalmente por predadores; b) procesos teratológicos, tales como disfunciones genéticas o embriológicas; y c) patologías diversas, frecuentemente debidas a la infestación por parásitos.

Las malformaciones en los trilobites se han documentado desde el Cámbrico Inferior hasta el Carbonífero, y el número elevado de casos observados en trilobites cámbricos y ordovícicos se correlaciona únicamente con el gran apogeo que alcanzó el grupo en ambos periodos. En este sentido, la disminución paulatina del registro de malformaciones en trilobites devónicos, silúricos y carboníferos, está siempre en relación con la abundancia relativa del grupo, y no tiene que ver con un incremento en las presiones biológicas que impidieran la supervivencia de los individuos anormales.

El trabajo de síntesis más completo sobre malformaciones en trilobites se debe a Owen (1985). En él se recogen 224 casos documentados con al menos una 


\section{CEFALÓN TÓRAX PIGIDIO TOTAL}

$\begin{array}{lrrrr}\text { CÁMBRICO } & 16 & 29 & 26 & 71 \\ \text { ORDOVÍ́CICO } & 44 & 14 & 34 & 92 \\ \text { SILÚRICO } & 7 & 6 & 34 & 47 \\ \text { DEVÓNICO } & 17 & 4 & 41 & 62 \\ \text { CARBONÍFERO } & - & - & 9 & 9 \\ \text { TOTAL } & 84 & 53 & 144 & 281\end{array}$

Figura 1. Número de anormalidades exoesqueléticas detectadas en los diferentes tagmas de los trilobites a nivel mundial. Datos tomados de Owen (1985) y modificados con la información aportada por Gandl (1972), Petr (1981, 1983), Snajdr (1985, 1990), Conway Morris y Jenkins (1985), Jell (1989), Babcock (1993), Archinal (1994), Owen y Tilsley (1996), Vokac (1996) y el presente trabajo.

tara en su morfología. En la Figura 1 se ha actualizado el número de citas, de las cuales 84 corresponden a daños detectados en la región cefálica, 53 en la torácica y 144 sobre el pigidio. Si tenemos en cuenta la repartición de dichas afecciones a lo largo del tiempo y cuales de ellas son las más numerosas, observamos que en primer lugar destacan las malformaciones cefálicas durante el Ordovícico, con 44 casos, seguidas por las que se encuentran sobre los pigidios durante el Devónico con 41, y el Ordovícico y Silúrico, que presentan cada uno de ellos otros 34 casos para esta misma parte corporal.

El registro de trilobites en la Península Ibérica es amplio y diverso en el Cámbrico, Ordovícico y Devónico, en tanto que resulta mucho más restringido en el Silúrico (por circunstancias paleogeográficas y paleoecológicas) y en el Carbonífero (con los trilobites ya en declive). No obstante esta abundancia, sólo hay cuatro citas sobre malformaciones documentadas en la bibliografía. La primera de ellas concierne a una patología sobre la glabela de Placoparia (Coplacoparia) borni Hammann, del Ordovícico de Ciudad Real (Hammann, 1971). Otras dos citas corresponden a una teratología en un pigidio de Asteropyginae del Devónico de la Cordillera Ibérica (Gandl, 1972), y a un pigidio de scutélido del Devónico de León, determinado por Smeenk (1983) como Platyscutellum sp., y que revisamos aquí. La última cita sobre patologías en trilobites de España se realiza en un trabajo (Isidro et al., 1996) en el que junto a.nueve formas teratológicas de mamíferos del Mioceno y Pleistoceno de España, se estudian dos ejemplares de trilobites con malformaciones, procedentes de la colección de S. Vela. De estos dos trilobites, uno de ellos corresponde a un Asteropyginae procedente de Canadá y por tanto no lo consideramos aquí. El otro ejemplar es una forma de Isabelinia glabrata (Salter) que procede del Ordovícico de Navas de Estena en la provincia de Ciudad Real; este ejemplar presenta la parte pleural izquierda de los cuatro segmentos anteriores de un tamaño menor que el normal en esta especie, y además distalmente muestran una morfología anómala. Posiblemente, como indican los autores del trabajo, el ataque de un predador pudo haber sido la causa de esa malformación. También en el resto del suroeste de Europa, el estudio de las malformaciones de los trilobites ha sido tratado muy poco y son muy escasas las citas en la literatura paleontológica. Entre ellas destaca la anomalía torácica observada en un ejemplar de Placoparia del Ordovícico armoricano, descrita por Henry (1980, lám. 6, fig. 1).

En este trabajo presentamos dos casos adicionales de malformaciones observadas en trilobites españoles, y revisamos el pigidio figurado por Smeenk (1983).

\section{DESCRIPCIÓN E INTERPRETACIÓN DE LAS MALFORMACIONES}

\author{
Asaphellus toledanus (Gil Cid) (Figs. 2A-B)
}

Descripción: El primer caso estudiado corresponde a un ejemplar completo de Asaphellus toledanus, del que se conserva el cefalón, el tórax y un fragmento del pigidio. Procede de las pizarras ferruginosas del Oretaniense inferior de Navas de Estena (Ciudad Real), y pertenece a la colección de José Martín Roldán, quien nos ha facilitado su estudio.

En vista dorsal se aprecia una malformación en la librigena derecha, con reducción ostensible del tamaño de la punta genal e irregularidades en el margen lateral del cefalón (Fig. 1a). La porción inmediatamente anterior al ángulo genal presenta un perfil cóncavo, con desarrollo de un reborde engrosado (Fig. 1b); la superficie de la librigena a la misma altura presenta también una depresión irregular. En posición más anterior se observa una segunda muesca en el margen lateral del cefalón, situada a nivel del extremo posterior de la superficie visual.

Interpretación: Por comparación con casos similares registrados en numerosos trilobites, la malformación analizada corresponde a una lesión traumática seguida de regeneración parcial. Aunque las proyecciones espinosas del caparazón representan las áreas más susceptibles de rotura accidental en los trilobites, la base de las puntas genales de Asaphellus toledanus es lo suficientemente robusta como para desestimar esta hipótesis, máxime en un fondo cenagoso y con escasa energía. En este sentido, las puntas genales son muy gruesas y la librigena posee un amplio borde ventral (Rábano, 1989). La otra posibilidad es que el daño fuese provocado por un predador, que desgajó buena parte de la librigena a partir del margen lateral. Éste muestra actualmente indicios de dos incisiones redondeadas, la posterior de las cuales presenta una callosidad marginal.

Con posterioridad a la rotura del caparazón, el animal sobrevivió y desarrolló una regeneración parcial hiperplásica de la zona afectada. Ésta se produjo a lo largo de diferentes fases de muda, atenuándose y cicatrizándose el contorno de las incisiones traumáticas, y llegándose incluso a una regeneración incipiente de la punta genal (Fig. 2b). Casos parecidos de regeneración 

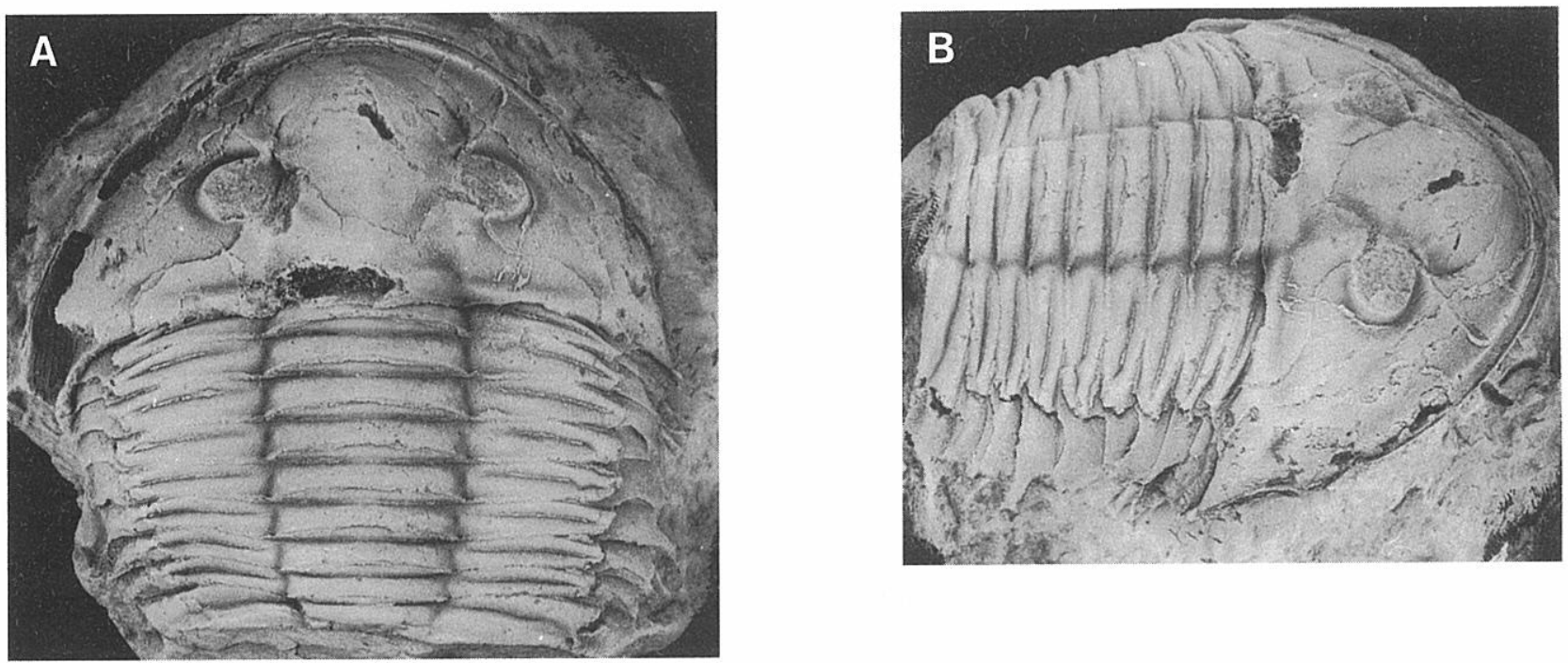

Figura 2. Asaphellus toledanus (Gil Cid), del Ordovícico Medio (Oretaniense inferior) de Navas de Estena Ciudad Real (Col. J. Martín Roldán). A, Vista dorsal. B, Vista lateral para apreciar la librigena derecha. Ambas x 1,1.

han sido descritos en Ceraurinella (hipostoma: Ludvigsen, 1977), Ogygopsis (pleuras torácicas: Rudkin, 1979), trinucleidos (Owen, 1983) y en Cedaria (con regeneración de la punta genal: Babcock, 1993).

En cuanto al posible causante de la lesión, las evidencias disponibles acerca de los predadores de los trilobites en el Ordovícico (Signor y Brett, 1984), apuntan a diversos artrópodos (euryptéridos, filocáridos), cefalópodos, asterozoos e incluso celentéreos (Alpert y Moore, 1975). Entre todos ellos nos inclinamos a pensar que el presente traumatismo fue causado por el ataque de un nautiloideo. El razonamiento se hace en primer lugar por exclusión, dado que euryptéridos y filocáridos son fósiles muy raros a partir del Ordovícico Superior centroibérico, y los únicos ofiuroideos contemporáneos (Palaeura neglecta hispanica Smith) no podrían producir, en vida del trilobites, una lesión de tal magnitud. Sin embargo, los nautiloideos ortoconos son fósiles frecuentes en las Capas con Tristani, donde algunos endocerátidos del Oretaniense inferior podrían superar el metro de longitud. En los bordes de muchas conchas de bivalvos y gasterópodos se reconocen incisiones triangulares y fragmentación, interpretables como marcas de predación de nautiloideos (GutiérrezMarco, com. pers.). Por ello es probable que el traumatismo estudiado en Asaphellus toledanus tenga un mismo origen.

Según Babcock (1993), el emplazamiento de la malformación en el lado derecho del trilobites podría no ser casual, y según su hipótesis podría deberse a la combinación de un comportamiento evasivo lateralizado desarrollado por los trilobites, y de una tendencia al ataque lateral por parte de sus predadores.

\section{Platyscutellum castroi (Mallada) (Fig. 3)}

Descripción: Pigidio MGM-470-D, Devónico Inferior, Formación La Vid, Colle (León). Este segundo ejemplo corresponde a un pigidio de Platyscutellum castroi que presenta unas dimensiones de $36 \mathrm{~mm}$ de largo y $51 \mathrm{~mm}$ de ancho, que entran dentro del margen de variabilidad intraespecífica de los individuos adultos que se encuentran en la misma localidad. La colección de $P$. castroi procedente de Colle y depositada en el Museo Geominero, consta de otros 32 ejemplares no patológicos, revisados recientemente por Arbizu et al. (1996).

El pigidio muestra sobre la tercera costilla del campo pleural derecho una malformación hacia su mitad abaxial, que limita primero su crecimiento en anchura, y luego la hace desaparecer abruptamente. La pérdida de relieve de esa zona produce una depresión ornamentada por algunos vestigios de crestas en terraza. La cuarta costilla, a su vez, está intercalada anormalmente entre la tercera y la quinta, en vez de arrancar directamente del surco dorsal del pigidio como es común en la especie, y se observa también en el flanco opuesto del mismo ejemplar (Fig. 3).

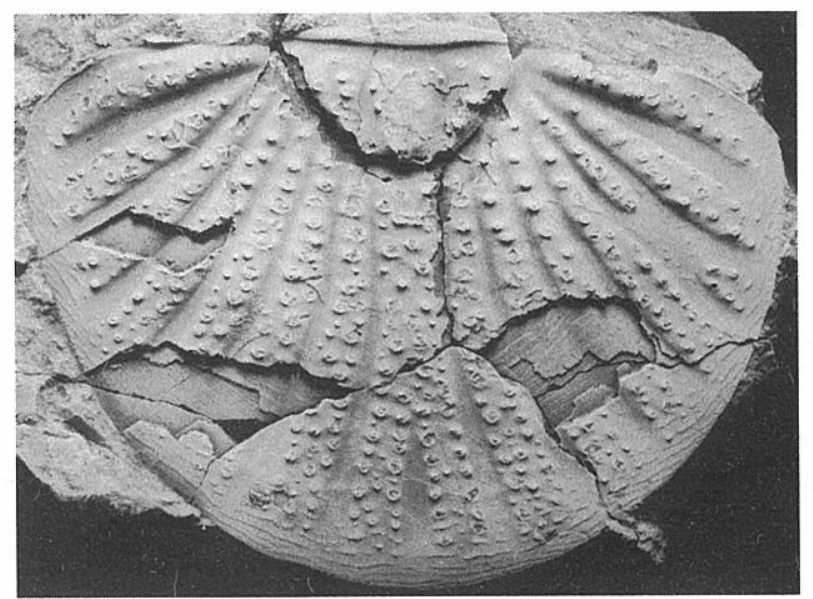

Figura 3. Platyscutellum castroi (Mallada), del Devónico Inferior (Emsiense inferior) de Colle (León). Pigidio MGM-470-D, x 1,5. 


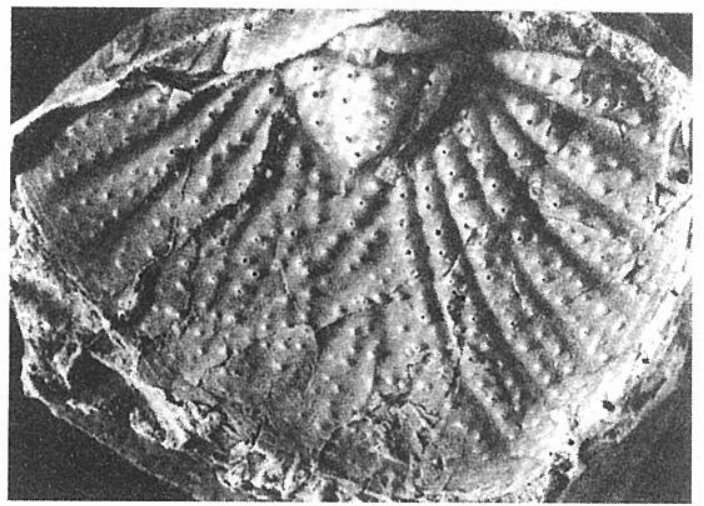

Figura 4. Platyscutellum castroi (Mallada), del Devónico Inferior (Emsiense inferior) de Colle (León). Molde en plástico del pigidio RGM 339529, x 2,6 .

Interpretación: Por comparación con otras malformaciones semejantes documentadas en scutélidos, la deformación que aquí nos ocupa pudo ser causada por la actividad de un parásito. Se ha descartado la posibilidad de que hubiera sido producida por un depredador y que el individuo hubiera restaurado prácticamente todo su exoesqueleto excepto esa zona. En primer lugar, por la restricción de la "lesión" a un área tan limitada del campo pleural y, en segundo lugar, por la ausencia de afectación de la misma a sucesivas posiciones del borde lateral del pigidio.

En cualquier caso, las malformaciones de origen parasitario conocidas en Scutelluidae (Snajdr, 1990) conducen normalmente a una alteración mucho más importante en la morfología que la observada en el ejemplar español. En este sentido, la única alternativa para la presente malformación es que represente un estado inicial de una pequeña infestación, que fue superada exitosamente por el animal al producirse en una fase relativamente avanzada de la ontogenia. A lo largo de mudas sucesivas, se manifestaron de nuevo los caracteres morfogénicos, con excepción de la pequeña área donde tuvo lugar la afectación. Esta se refiere a dos caracteres: el primero en ocurrir fue un retardo en la aparición de la cuarta costilla pleural, que aparece intercalada entre la tercera y la quinta. Paralelamente, la tercera costilla no experimenta un aumento progresivo en anchura y resulta omitida en determinado momento del crecimiento. Así, tras una interrupción de la misma en un espacio vacío de $6 \mathrm{~mm}$ de longitud, coincidente con uno o varios periodos de muda, la tercera costilla reapareció para configurar un extremo distal bifurcado de apariencia y extensión completamente normal.

\section{Platyscutellum castroi (Mallada) (Fig. 4)}

Descripción: Se trata del pigidio figurado por Smeenk (1983: lám. 29, fig. 6), procedente también de la localidad de Colle (Formación La Vid, Devónico Inferior). Está depositado en el Rijksmuseum van Geologie en Mineralogie (Leiden, Holanda) con el $n^{\circ}$
RGM 339529. Una copia en plástico se conserva en el Museo Geominero de Madrid.

El pigidio se encuentra muy afectado en la parte adaxial del campo pleural izquierdo, en la costilla media y parte de la séptima del campo pleural derecho. Las costillas tercera a séptima izquierdas, y la media, han desaparecido como tales; en su lugar se observa una disposición caótica de hileras de tubérculos, habiendo perdido en gran parte su tendencia a la disposición radial. La primera costilla del lado izquierdo falta, al igual que la porción adaxial de la segunda y el ápice inicial de la tercera. Estas dos últimas no presentan una deformación notable, aunque hacia la región abaxial su relieve es menor del que le correspondería, y los surcos están también menos marcados. El contorno pigidial, carácter importante en la determinación genérica, muestra también un trazado irregular, débilmente arqueado hasta la prolongación de la séptima costilla del campo pleural derecho, que no es propio de ninguno de los géneros de Scutelluidae.

Interpretación: Como en el pigidio descrito anteriormente, esta malformación fue el resultado de una infestación parasitaria, aunque con un grado de desarrollo diferente. El estado infeccioso es muy avanzado, dando lugar a lo que en estos casos se denomina una malformación extrema, producida en poco tiempo por un agente muy virulento, o bien el parásito pudo ser menos dañino pero haber actuado durante más tiempo para producir el mismo efecto.

En este caso nos inclinamos por la primera posibilidad, ya que el ejemplar presenta un tamaño menor que la mayoría de los pigidios adultos de esta especie que se encuentran en los mismos niveles de procedencia. Así, el agente que lo infestó solo pudo actuar durante un lapso temporal menor que si hubiera sido sobre un ejemplar de mayor tamaño, y por lo tanto más viejo. De todas formas, aún cabe la duda de que esa misma afección fuera la causa de un desarrollo deficiente, y pudieramos estar también frente a un caso de raquitismo a la vez que de malformación.

\section{CONCLUSIONES}

Las consideraciones que se han tenido en cuenta para la interpretación del origen de cada una de las malformaciones estudiadas no permiten asegurar, de una manera absoluta, que éstas no sean el resultado de una teratología genética o embriológica, o bien el resultado de un accidente fortuito durante la muda. Sin embargo, y por comparación con casos documentados en trabajos previos, se pueden desestimar estas posibilidades. La malformación del cefalón del asáfido A. toledanus debió ser causada por un depredador, probablemente un nautiloideo. En el caso de los dos pigidios de $P$. castroi, fueron patologías producto de infestaciones parasitarias. En el primero de los casos parece que corresponde a una fase incial, mientras que el segundo representa ya un estadio muy avanzado. 
Los tres ejemplos aquí presentados contribuyen a incrementar el conocimiento sobre malformaciones en trilobites, describiendo e interpretando tres casos registrados en ejemplares españoles.

\section{AGRADECIMIENTOS}

Los autores agradecen a José Martín Roldán el préstamo del ejemplar de A. toledanus para su estudio, así como al Dr. Z. Smeenk (Leiden) el habernos enviado el molde del pigidio teratológico de $P$. castroi por él ilustrado. Uly Martín (Universidad Complutense) realizó las fotografías. Este trabajo es una contribución al proyecto 05P/006/1996 de la Dirección General de Investigación de la Comunidad de Madrid.

\section{BIBLIOGRAFÍA}

Alpert, S.P. and Moore, J.N. 1975. Lower Cambrian trace fossil evidence for predation on trilobites. Lethaia, $\mathbf{8}$, 223-230.

Arbizu, M., Rábano, I. y Truyols, J. 1996. Trilobites del Museo Geominero. III. Las colecciones antiguas del Devónico de la Cordillera Cantábrica. Boletín Geológico y Minero, 107, 3-13.

Archinal, A. 1994. Zur Morphologie, Systematik, Stratigraphie und Ökologie der Gattung Scutellum (Trilobita; Devon). Senckenbergiana lethaea, 74 (1/2), 291-324.

Babcock, L.E. 1993. Trilobite malformations and the fossil record of behavioral assymetry. Journal of Paleontology, 67, 217-229.

Conway Morris, S. and Jenkins, R.J.F. 1985. Healed injuries in Early Cambrian trilobites from South Australia. Alcheringa, 9, 166-177.

Gandl, J. 1972. Die Acastavinae und Asteropyginae (Trilobita) Keltiberiens (NE Spanien). Abhandlungen der senckenbergischen naturforschenden Gesellschaft, 530, 1-184.

Hammann, W. 1971. Die Placopariinae (Trilobita, Cheirurina, Ordovizium). Senckenbergiana lethaea, 52, 53-75.

Henry, J.-L. 1980. Trilobites ordoviciens du Massif Armoricain. Mémoires de la Société géologique et minéralogique de Bretagne, 22, 1-250.

Isidro, A., Malgosa, A., Belinchón, M., Vela, S., Alcalá, M., Dieguez, C., Castellana, C. and Fernández, S. 1996. Zoo-
Paleopathology from Spain: A discussion of some cases. Journal of Paleopathology, 8 (3), 143-152.

Jell, P.A. 1989. Some aberrant exoskeletons from fossil and living arthropods. Memoirs of the Queensland Museum, 27, 491-498.

Ludvigsen, R. 1977. Rapid repair of traumatic injury by an Ordovician trilobite. Lethaia, 10, 205-207.

Owen, A.W. 1983. Abnormal cephalic fringes in the Trinucleidae and Harpetidae (Trilobita). Special Papers in Palaeontology, 30, 241-247.

Owen, A.W. 1985. Trilobite abnormalities. Transactions of the Royal Society of Edinburgh, Earth Sciences, 76, 255272.

Owen, A.W. and Tilsley, J.W. 1996. An abnormal pygidium of the trilobite Brachymetopus ornatus Woodward from the Lower Carboniferous of Derbyshire. Geological Journal, 31, 389-392.

Petr, V. 1981. Anomálni pygidium trilobita Platyscutellum formosum formosum (Barrande, 1846) ve sbírkách Okresního muzea v Beroune. Cesky Kras, 6, 44-46.

Petr, V. 1983. Teratologické pygidium trilobita Radioscutellum intermixtum (Hawle et Corda, 1847) z konepruskych vapencu ve sbírkách Okresního muzea v Beroune. Cesky Kras, 8, 56-59.

Rábano, I. 1989. Trilobites del Ordovícico Medio del sector meridional de la zona Centroibérica española. Parte II. Agnostina y Asaphina. Boletín Geológico y Minero, 100, 541-609.

Rudkin, D.M. 1979. Healed injuries in Ogygopsis klotzi (Trilobita) from the Middle Cambrian of British Columbia. Royal Ontario Museum, Life Sciences Occasional Paper, 32, 1-8.

Signor, P.W. and Brett, C.E. 1984. The mid-Paleozoic precursor for the Mesozoic marine revolution. Paleobiology, 10, 229-245.

Smeenk, Z. 1983. Devonian trilobites of the Southern Cantabrian Mountains (Northern Spain) with a systematic description of the Asteropyginae. Leidse Geologische Mededelingen, 52, 383-511.

Snajdr, M. 1985. Anomalous exoskeletons of Bohemian encrinurine trilobites. Vestník Ústredního ústavu geologického, 60, 303-306.

Snajdr, M. 1990. Five extremely malformed scutelluid pygidia (Styginidae, Trilobita). Vestník Ústredního ústavu geologického, 65, 115-118.

Vokac, V. 1996. O nekolika abnormalitách trilobitovych exoskeletonu ze stredoceského starsiho paleozoika. Palaeontologia Bohemiae, 1996, 20-22. 